\title{
1 Problems using ordinal traits with continuous measures of functional diversity
}

2

3

4

\author{
Matt Davis $1,2,3,4$
}

${ }^{1}$ Natural History Museum of Los Angeles County, Los Angeles, CA, USA.

${ }^{2}$ Aarhus University, Department of Bioscience, Aarhus, Denmark.

${ }^{3}$ Yale University, Department of Geology and Geophysics, New Haven, CT, USA.

${ }^{4}$ Smithsonian Institution, National Museum of Natural History, Department of Paleobiology,

Washington, DC, USA. 
Abstract

Continuous indices of functional diversity are popular in studies examining community

12 structure and ecosystem function across a wide range of subfields from paleontology to range

13 management. These indices were designed to replace the use of more arbitrary, discrete

14 functional groups or guilds; however, the effect of typical methodological decisions on these new

15 continuous measures has not been fully investigated. To test the effect of using ordinal traits in

16 functional diversity analysis, I first calculated relative functional diversity index values in real

17 plant communities with real continuous trait data and Euclidean distances. I then compared these

18 original values to "treatment" functional diversity index values obtained by discretizing the trait

19 data and using Gower's distance. Agreement between original and treatment values was highly

20 unpredictable and often abysmal. Small methodological choices, such as whether to treat a

21 functional trait as continuous (mm) or ordinal ("small", "medium", "large"), could completely

22 change a perceived functional diversity relationship along an environmental gradient. Some

23 parameter combinations returned results that were no better than random noise. Because simple

24 methodological choices can have such a large impact on continuous functional diversity indices,

25 it is ambiguous whether analyses using ordinal traits are actually measuring an underlying

26 functional diversity relationship between communities or just reflecting the arbitrary parameter

27 choices of researchers.

29 Key-words: functional diversity, Gower's distance, ordinal traits, continuous, discrete,

30 dendrogram, convex hull, multidimensional, functional richness, functional evenness 
Introduction

Although often described as a burgeoning new subfield (Petchey and Gaston 2006), or even a bandwagon (McGill 2015), the study of functional trait diversity, the range and value of species or organismal traits that effect ecosystem functioning (Tilman 2001), has long been an integral part of ecological theory and practice previously investigated through morphospace or

37 functional groups and guilds (e.g. Holmes et al. 1979, Van Valkenburgh 1988). Despite mixed evidence (Stevens and Tello 2014), functional diversity is thought to represent a unique aspect of biodiversity (Devictor et al. 2010) that could potentially explain ecosystem function, species coexistence, and community assembly better than taxonomic richness or phylogenetic diversity

41 alone (Tilman et al. 1997, Hulot et al. 2000, Díaz and Cabido 2001, Loreau et al. 2001,

42 Heemsbergen et al. 2004, Hooper et al. 2005, Mason et al. 2005, Pavoine and Bonnan 2011).

43 Similar to phylogenetic diversity, which moved from subjectively defined taxonomic units to

44 increasingly sophisticated statistical methods of tree discovery (Felsenstein 2004, Redding et al.

45 2014), functional diversity has changed from the census of discrete, arbitrarily defined functional

46 guilds (e.g. large carnivore, medium carnivore) (Fonseca and Ganade 2001) to complex

47 measures of multidimensional functional space (Mouchet et al. 2010, Schleuter et al. 2010) that

48 can be used to model future structural changes in ecosystems (Barbet-Massin and Jetz 2015) or

49 triage species endangered by climate change (Buisson et al. 2013).

Central to this transformation was the view that a priori discrete functional groups,

51 although frequently used, were not based on objective criteria (Petchey et al. 2009),

52 overestimated redundancy by lumping together heterogeneous species (Fonseca and Ganade

53 2001), and often provided no predictive power over random classifications (Wright et al. 2005).

54 New measures of functional diversity had to be continuous and relatively independent of 
55 arbitrary researcher decisions (Petchey et al. 2004). Though others had used both multidimensional functional space and functional dendrograms before to examine community

57 structure (e.g. Holmes et al. 1979), the theoretical framework for these modern indices was laid

58 by Rosenfeld (2002) who formally defined functional space as a hypervolume delineated by axes

59 of functional traits or processes (e.g. $\mathrm{CO} 2$ production, $\mathrm{N}$ fixation, etc.) analogous to

60 Hutchinson's (1957) n-dimensional niche defined by environmental axes. Using this functional

61 space, Villéger et al. (2008) developed a general methodology for calculating continuous

62 functional diversity indices that was expanded on and operationalized by Laliberté and Legendre

63 (2010) into the now popular R software package "FD". First, researchers measure ostensibly

64 functionally important traits of taxa (typically species) and after standardization, use these traits

65 to generate a species-species dissimilarity matrix. These interspecies distances are subjected to a

66 principle coordinates analysis $(\mathrm{PCoA})$ that places species in a multidimensional Euclidean trait

67 space where a range of functional index values can be calculated on the species' new traits (the

68 PCoA axes). This methodological framework is also used to construct the popular dendrogram-

69 based functional richness index (FD) developed by Petchey and Gaston $(2002,2007)$ and

70 modified by Podani and Scherma (2006) except that, instead of PCoA, the species-species

71 distance matrix is run through a sorting algorithm such as unweighted pair-group clustering

72 method using arithmetic averages (UPGMA) to generate a functional tree with species as tips.

73 Calculating these Euclidean distances between species is straightforward with continuous

74 traits measured on interval and ratio-scales such as body temperature $\left({ }^{\circ} \mathrm{C}\right)$ or leaf area $(\mathrm{mm} 2)$,

75 respectively, but some continuous traits such as flowering time (circular variable), or percentage

76 of diet from different feeding categories (fuzzy variable) cannot immediately be compared using

77 Euclidean distances without some kind of transformation. Many traits are discrete, such as 
78 presence or absence of spines (binary variable), growth form (multistate nominal variable), or

79 height class (ordinal variable), and aside from binary variables, they cannot be readily converted

80 into Euclidean distances. This is why all developers of this common functional diversity

81 methodological framework (Podani and Schmera 2006, Petchey and Gaston 2007, Villéger et al.

82 2008, Laliberté and Legendre 2010) suggest that researchers use Gower's general coefficient of

83 similarity (Gower 1971) to calculate distances between taxa when considering variables of mixed

84 types.

85 Gower originally developed his coefficient for quantitative taxonomy (Pavoine et al.

86 2009) but its Euclidean properties and adaptability made it widely popular and useful in fields as

87 disparate as retail science (Churchill et al. 1975) and dermatology (Katrina et al. 1985). Gower

88 calculated the similarity $S_{j k}$ of two taxa (typically species) $j$ and $k$ as,

90 Eqn. 1

$$
S_{j k}=\sum_{i=1}^{n} s_{i j k} \delta_{i j k} w_{i} / \sum_{i=1}^{n} \delta_{i j k} w_{i}
$$

93 where $n$ is the number of traits examined, $w_{i}$ is the variable weight, $s_{i j k}$ is the similarity (ranging

94 from 0-1) between taxa $j$ and $k$ calculated on trait $i$, and $\delta_{i}=0$ if the $i$ th trait value is missing for

95 one or both taxa and $\delta_{i}=1$ if both taxa posses the value and can be compared. Gower defined the

96 similarity $s_{i j k}$ individually for continuous, nominal, and binary variables (Gower 1971), with later

97 authors defining extensions for ordinal variables (Podani 1999); multichoice nominal and

98 associated-binary variables (Podani and Schmera 2007); and circular, fuzzy, and correlated-

99 proportion variables (Pavoine et al. 2009). In the simplest case where there are no missing values 
100 and all traits are weighted equally, Gower's coefficient is the average of these different

101 similarities:

103 Eqn. 2

$$
S_{j k}=\sum_{i=1}^{n} s_{i j k} / n
$$

For the rest of this paper, Gower's coefficient will be treated as a distance metric $D_{j k}=1-S_{j k}$.

Functional diversity studies implicitly assume that there is some true quantity "functional diversity" that measures the distribution of organisms' contributions to ecosystem functioning

109 and that this quantity can be estimated by indices calculated from several measurable traits of

110 taxa. However, if methodological choices such as the inclusion of categorical traits requiring

111 Gower's distance greatly affect relative functional index values between communities, it is

112 doubtful that these indices actually estimate true functional diversity. Continuous measures of

113 functional diversity could suffer from the same types of arbitrary decisions by researchers as the

114 functional guilds they were meant to replace.

115 Although there is no universally "correct" distance metric, we often intuitively

116 conceptualize functional niche space as a realm measurable by Euclidean distances (Figure 1).

117 Hutchinson (Hutchinson 1957) originally defined his n-dimensional hypervolume by

118 environmental variables, “... which can be measured along ordinary rectangular coordinates” but

119 admitted that this unrealistic assumption was a limitation of his concept. Petchey and Gaston

120 (2007) suggest that this distinction may be trivial in practice as they arrived at very similar

121 functional index values whether using Gower's distance or (incorrectly) using Euclidean distance 122 on datasets with mixed variable types. The users' manual for the FDiversity software package 
123 (Casanoves et al. 2008) also advises that using Gower's distance may not change results.

124 However, it is clear that using different variable types, and by extension different distance

125 metrics, could produce large downstream changes among analyses. Different variable types lead

126 to specific shapes of point clouds in multidimensional space. Continuous variables produce a

127 continuous line of points but circular variables lead to arcs or circles in space and nominal

128 variables separate points into distinct groups located at the vertices of regular polygons (Pavoine

129 et al. 2009).

130 Both Gower's and Euclidean distances can handle continuous and binary variables, but to

131 measure the impact of using other types of discrete traits, and by extension Gower's distance, we

132 must consider ordinal characters. Nominal traits have no equivalent in Euclidean space: there are

133 no lines on a ruler for "wind dispersed" and "animal dispersed" by which to measure different

134 seeds. However, ordinal traits such as "small", "medium", and "large" typically represent some

135 underlying, measurable continuous distribution for which we lack complete data. By taking a

136 known continuous distribution and discretizing it into ordered levels, we can investigate how

137 inclusion of discrete characters alters relative functional index values between communities. If

138 these indices are accurately estimating some true underlying value of functional diversity,

139 continuous traits and ordinal traits reflecting the same distribution should produce similar results.

140 If index values are wildly different, then we must admit that some measures of functional

141 diversity reflect nothing more than arbitrary methodological choices.

143 Methods

144 In order to test the effect of discretization and Gower's distance on functional diversity

145 values, I first calculated functional diversity indices with continuous trait data and Euclidean 
146 distances following the standard methodology of Laliberté and Legendre (2010). For simplicity,

147 these data, and results generated from them are hereafter referred to as the "original"

148 data/distances/values. I then artificially discretized those continuous trait data and reran analyses

149 using several versions of Gower's distance. For simplicity, those data and results are referred to

150 as the "treatment" data/distances/values. By comparing relative functional diversity index values

151 between the original and treatment methods, we can see the impact that discrete traits and

152 Gower's distance have on functional diversity analyses (Figure 2).

154 dataset (Laliberté et al. 2008) represents a 2007 census of a series of experimental plots in New

155 Zealand measuring the effect of grazing and fertilizer on plant functional diversity. The

156 "Roadside" dataset (Thompson et al. 2010) represents a series of small quadrats measuring plant

157 species richness every year between 1958 and 2003 along a stretch of road in England. Datasets

158 were trimmed to only continuous traits and any species missing data were removed. Percentage

159 occupancy data were converted to presence/absence by counting all species that occurred in a

160 site. After data conditioning, the Tussock dataset had 41 species with eight traits in 30

161 communities. The Roadside dataset had 75 species with seven traits in 325 communities (plots

162 measured at different time points). The use of these data should in no way be viewed as a

163 critique on their validity, rather the opposite. I used them because they considered a large

164 number of quantitative traits and their authors graciously made them available.

165 To create the original functional diversity index values to compare to the discretized

166 treatment data, I first took the Euclidean distance between species based on their continuous

167 traits. Trait values must be standardized so results are not driven by units of measurement (Lepš

168 et al. 2006). I tested the effects of two popular methods of standardization: the defaults for 
169 calculating Euclidean and Gower's distance in R version 3.2.2 (R Development Core Team

170 2015). In one set of simulations, continuous traits were standardized by the distance function

171 daisy in the R package "cluster" (Maechler et al. 2015) that subtracts the mean of the trait from

172 each value and then divides by the original average distance to the mean for that trait. In the

173 other set of simulations, continuous trait values were divided by trait ranges. Gower (1971)

174 argued that standardizing by trait range made more sense as it could be easily measured on the

175 sample or calculated from theoretical limits (e.g. the minimum and maximum body mass

176 possible for mammals) and it was more immediately interpretable compared to the standard

177 deviation.

Continuous data were discretized into three to ten ordered levels either along absolute

179 splits where the trait range was equally divided or by quantile splits where an equal number of

180 species were included in each level. Although continuous data can be discretized into two

181 ordered levels, in practice they would more likely be dichotomized into binary variables and

182 treated as such. Note that because R collapses levels unoccupied by data points, the given

183 number of levels should be taken as a maximum. For example, a trait split equally along its range

184 into ordered levels 1, 2, 3, and 4 could be collapsed into ordered levels 1, 2, and 3 (formerly 4) if

185 no species occupied the original level 3. Functional trait data were discretized one trait at a time

186 until all traits were discretized into ordered levels. Each possible combination of traits was

187 considered (e.g. the two discretized traits could be the 1 st and 2 nd traits, or the 1 st and 3 rd traits,

188 or the 2 nd and 3 rd traits, etc.). It may seem unrealistic to have a functional dataset where the

189 majority of traits are ordinal or where ordinal traits take more than a few levels but both

190 scenarios are frequent in the literature. For example, 19 out of the 20 functional traits used by 
191 Shütz and Schulze (2015) for urban birds were ordinal. In the standardized list of plant traits

192 (Cornelissen et al. 2003), flammability is an ordinal trait with 41 levels.

193 There are several different ways to calculate dissimilarities with ordinal traits (Table 1).

194 Gower (1971) originally lacked a formal measure for such traits, treating ordinal scores as

195 continuous data by taking the Manhattan distance standardized by the range of values (Table 1,

196 Continuous), although one could also take the Euclidean distance of these values (Table 1,

197 Euclidean). Both methods are mathematically dubious; technically, the differences between

198 ordinal traits are not interpretable (Podani 1999). Sneath and Sokal (1973) proposed that ordinal

199 data should be treated as unranked categories despite a potential loss of information (Table 1,

200 Categorical). Podani (1999) created two formal extensions of Gower's distance. One, metric,

201 took the Manhattan distance between ranks and standardized by the range of ranks (Table 1,

202 Metric). The other, nonmetric, calculated the number of interchanges in taxon rank order to

203 move a species from one rank to another and standardized by the total number of interchanges

204 possible (Table 1, Podani). This is likely the most commonly used method as it is the default for

205 the popular R program dbFD used in the functional diversity analysis package "FD" (Laliberté

206 and Legendre 2010). Pavoine et al. (2009) proposed another measure analogous to Podani's

207 (1999) metric equation by taking the Euclidean distance of ranks standardized by the range of

208 ranks, although they took the square root of any resulting distances to lend them Euclidean

209 properties (Gower 1971) (Table 1, Pavoine). During this study, I discovered a bug in the code

210 for the Gower's distance function dist.ktab (Pavoine et al. 2009) in the R package "ade4"

211 (Chessel et al. 2004) that can effectively randomize a matrix of ordinal trait data. To avoid this

212 bug, all ordinal traits should be designated as "numeric" class variables in R, not "ordered" 
213 "factor" class as one would assume. Any study using this function in "ade4" version 1.7-4 or

214 earlier, before a clarifying warning was added, should be reevaluated.

215 Species-species distance matrices were calculated by Euclidean distances on the original

216 continuous traits or Gower's distances on the discretized traits using the methods for ordinal data

217 described above. Euclidean distances were also calculated on discretized traits as though they

218 were continuous (Table 1, Euclidean). Both original and treatment species-species distance

219 matrices were used to calculate six popular indices of functional diversity (Figure 2). With the

220 exception of Petchey and Gaston's (2007) modified dendrogram-based functional richness (FD),

221 they can all be generated with the R package "FD" (Laliberté and Legendre 2010). FD (Petchey

222 and Gaston 2007) and FRic (Villéger et al. 2008) measure the volume of functional trait space

223 occupied. FDiv (Villéger et al. 2008) and FDis (Laliberté and Legendre 2010) measure species

224 distributions relative to the center of trait space. RaoQ (Botta-Dukát 2005) measures how closely

225 species are packed into trait space, and FEve (Villéger et al. 2008) measures how regularly they

226 are packed.

227 To test how the inclusion of discrete characters affected relative functional diversity

228 values, I calculated the root mean squared error (RMSE) between original community functional

229 diversity index values and treatment index values (Figure 3). Unfortunately, a major

230 shortcoming of functional diversity analyses is that raw index values cannot typically be

231 compared between studies. They are informative only in their relative value within a dataset. For

232 this reason, all indices were standardized by the range of community values before being

233 compared to each other. This generates a conservative estimate of error as this standardization

234 increases the likelihood of a 1:1 relationship between original index values and treatment index

235 values compared to some typical ways of standardization, such as making FRic or FD 
community values proportional to the total convex hull volume or tree length of all species in the

237 dataset, respectively. However, this is probably the only fair way to compare errors between

238 indices, as given other standardization methods, different indices will tend to occupy different

239 proportions of the $0-1$ domain. For example, if average community species richness is low

240 compared to regional richness, standardizing by the regional convex hull volume will always

241 produce low community FRic values, and by extension, low RMSE between actual and modeled 242 values.

243 For perspective, these RMSE values were compared to RMSE values obtained from 244 perfect negative correlations to the original indices and the $95 \%$ confidence intervals of 100,000

245 simulations of random values compared to the original indices (Figure 3, Figure 4). Note that

246 the confidence intervals for random values are also very conservative. Depending on the

247 underlying structure of the original data, it is possible to find distributions of random values (e.g.

248 drawn from a normal distribution) that are statistically unrelated to the original indices, but still

249 return much lower RMSE values than samples drawn from the uniform distribution bounded

250 between 0 and 1 that was used to construct the confidence intervals. To determine the marginal

251 effect of each parameter on RMSE (Figure 5, Figure A 1), I used the partialPlot function on a

252 model generated from 1,001 regression trees in the R package randomForest (Liaw and Wiener

253 2002) under default parameters leaving a random $30 \%$ of the data out for testing. The importance

254 of different parameters for determining RMSE was found by measuring the decrease in the

255 residual sum of squared error in the regression tree when it was split on a given parameter. This

256 value was averaged across all 1,001 trees in the random forest. 
In general, the inclusion of discrete traits and the use of Gower's distance had a large

260 effect on functional diversity analyses, although the severity of this effect was highly variable

261 and not always expected. The specific effects of each parameter combination are likely to vary

262 from dataset to dataset, so readers are encouraged to focus on the general qualitative results,

263 rather than the exact values, found here. The model generated from the random forest fit the data

264 well, predicting the $30 \%$ left out for testing with a pseudo $\mathrm{R}^{2}$ of $\sim 0.93$ for both the Tussock and

265 Roadside datasets. The functional diversity index used was the most important parameter for

266 predicting RMSE, while number of discrete levels was the least important (Figure 5, Figure A

267 1). Within each parameter group, individual parameters varied in how much they affected

268 functional diversity values, although some indices performed relatively worse (FDiv), or better

269 (FD), than others in both datasets (Figure 5, Figure 6, Figure A 1, Figure A 2). Detailed graphs

270 of results for all parameter combinations on both datasets are included in Appendix 1.

$271 \quad$ For ordinal traits using the Podani method of Gower's distance, a common default

272 parameter combination, discretizing continuous data into more levels increases similarity

273 between original and treatment data as expected, but not monotonically (Figure 4). Depending

274 on the underlying distribution of the original continuous data, nine or ten levels may make

275 discretized treatment index values more different than only discretizing the data into three levels.

276 Increasing the number of discretized traits increases error in the RaoQ index value as expected,

277 but surprisingly only if the data have been discretized into seven to ten levels. Data discretized

278 into three to six levels actually produce index values more similar to original continuous values

279 as the number of the traits discretized increased. In the Roadside dataset, different Gower's

280 distance methods show large differences in RMSE, but only when the original continuous data

281 are discretized using absolute splits (Figure A 2). Different Gower's distance methods produce 
282 almost identical RMSE if traits are split along quantiles. And counterintuitively, far from losing

283 information, treating ordinal traits as unranked categories actually approximates the original

284 index values better than almost all other methods (Figure 5, Figure A 1).

\section{Discussion}

When comparing values from the treatment data to the original data (Figure 6, Figure A

2), certain results are expected. Treatment index values should match original index values

standardized by range better than those standardized by the daisy function because the treatment data are already standardized by range with Gower's distance. We would also expect that

291 increasing the number of traits discretized, or reducing the number of discrete levels, should

292 make treatment index values, on average, more different than original index values. Euclidean

293 distances are likely to return similar values on continuous and discretized traits compared to

294 distances using a more complicated rank interchange formula. Beyond these simple predictions,

295 however, it is hard to see any consistent pattern in the results except that small parameter

296 changes can have large and unpredictable results.

297 It should not be surprising that using different parameters and different methods for

298 calculating distances between species will produce different functional diversity index values.

299 Different methods represent different mathematical equations and ways of parsing data. Results

300 should be different. What is surprising is how large and unpredictable these differences are, and

301 how much they would impact empirical functional diversity analyses. For example, a researcher

302 following the typical workflow described above with continuous trait data standardized by the

303 daisy function would find that in the Tussock dataset, FDiv decreases with increased fertilizer

304 application up to $250 \mathrm{~kg}$ ha-1 $\mathrm{yr}-1$ before increasing again, adjusted $\mathrm{R}^{2}=0.59, \mathrm{p}<0.0001$ 
305 (Figure 7, red line). This researcher could then go on to hypothesize why increasing exogenous nutrient loads first drives species closer together in functional space before driving them back

307 apart. The results would surely be of great practical use for preserving ecosystem functions in or

308 near crop-dominated landscapes. However, consider a second researcher. This researcher uses

309 the same data except that two of the eight functional traits, leaf dry matter content and leaf

310 phosphorous concentration, are measured only as "Low", "Medium", or "High". However,

311 using typical workflows and choosing Podani’s (1999) metric method for Gower's distance on

312 ordinal traits, this researcher will find the exact opposite pattern (Figure 7, blue line): FDiv

313 increases up to $250 \mathrm{~kg}$ per ha per year and then decreases, adjusted $\mathrm{R}^{2}=0.55, \mathrm{p}<0.0001$. A third

314 researcher using identical methods and data as Researcher \#2, except that leaf nitrogen

315 concentration instead of leaf dry matter content is now "Low", "Medium", and "High" will find

316 yet another pattern: FDiv increases linearly with fertilizer loads, adjusted $\mathrm{R}^{2}=0.34, \mathrm{p}<0.001$

317 (Figure 7, purple line).

FDiv was admittedly the worst performing index considered, but this example using real

319 data and standard methods still raises doubts about the reliability of any functional diversity

320 analysis. If we can fundamentally change a perceived underlying functional diversity relationship

321 by discretizing one trait instead of another, then we have little hope of controlling for the varied

322 effects of different taxa, traits, methods, and study systems to arrive at general principles about

323 functional diversity. Gower's distance could be avoided by making Euclidean distances on

324 continuous data the gold standard for analysis. But if only continuous data are used, what effect

325 will removing likely non-random subsets of genuinely discrete traits (e.g., C3, C4, and CAM

326 pathways) have on functional diversity analyses? We desperately need more basic research into 
327 functional diversity methods and indices before we can begin to make meaningful interpretations

328 of natural patterns.

Of all the treatment parameter combinations, discretizing data at absolute splits and

330 taking Euclidean distance on the resulting ordered levels produced the greatest similarity to the

331 original index values. Although not optimal, using traits with four or more roughly equally

332 spaced ordered levels and Euclidean distance may be a workable stopgap for including discrete

333 characters in functional diversity analyses until we can devise a more robust methodology.

334 Treating ordinal values with Euclidean distance is technically improper mathematically, but all

335 continuous traits are actually discrete at some scale. Consider a researcher measuring plant

336 heights in a population of forbs ranging from around 3 to $95 \mathrm{~mm}$ tall; a ruler marked only by

337 centimeters would be a coarse but methodologically sound tool. Using ten ordered levels is

338 operationally no different. For functional diversity indices, Petchey and Gaston's (2007)

339 modified dendrogram-based FD may be the best choice at present, as it was the least sensitive to

340 parameter changes considered here, and is easily compared to phylogenetic diversity. However,

341 it unrealistically assumes hierarchical functional variation among taxa (Petchey and Gaston

342 2006) and is known to reflect methodological choices more when communities have similar

343 species richness (Poos et al. 2009).

344 No methodology will be perfect for all situations, and any methodology must be based on

345 sound statistical and biological arguments (Pavoine et al. 2009) that best suit the ecological

346 question at hand (Lepš et al. 2006). The measurement of distances between species considered

347 here is just one critical step in functional diversity analysis (Pavoine et al. 2009). Others (Lepš et

348 al. 2006, Lavorel et al. 2008) have shown how small methodological choices in data collection

349 can also have outsized impacts on functional diversity index values. New methods will have to 
acknowledge this uncertainty and be able to integrate across multiple parameter settings to

351 provide more robust measurements of functional diversity.

\section{Acknowledgements}

\section{References}

Barbet-Massin, M. and Jetz, W. 2015. The effect of range changes on the functional turnover, structure and diversity of bird assemblages under future climate scenarios. - Global Change

Botta-Dukát, Z. 2005. Rao's quadratic entropy as a measure of functional diversity based on multiple traits. - J Veg Sci 16: 533-540.

Buisson, L. et al. 2013. Toward a loss of functional diversity in stream fish assemblages under climate change. - Global Change Biol 19: 387-400.

Casanoves, F. et al. 2008. User manual FDiversity: statistical software for the analysis of functional diversity.

Champely, S. and Chessel, D. 2002. Measuring biological diversity using Euclidean metrics. -

Chessel, D. et al. 2004. The ade4 package-I: one-table methods. - R News 4: 5-10.

Churchill, G. A. et al. 1975. Should retail salespersons be similar to their customers? - Journal of $372 \quad$ Retailing 51:29-79.

373 Cornelissen, J. H. C. et al. 2003. A handbook of protocols for standardised and easy

Devictor, V. et al. 2010. Spatial mismatch and congruence between taxonomic, phylogenetic and functional diversity: the need for integrative conservation strategies in a changing world. Ecol. Lett. 13: 1030-1040.

Díaz, S. and Cabido, M. 2001. Vive la différence: plant functional diversity matters to ecosystem processes. - Trends Ecol. Evol. 16: 646-655. 
380 Felsenstein, J. 2004. Inferring Phylogenies. - Sinauer Associates.

381 Fonseca, C. R. and Ganade, G. 2001. Species functional redundancy, random extinctions and the stability of ecosystems. - J. Ecol. 89: 118-125.

Gower, J. C. 1971. A General Coefficient of Similarity and Some of Its Properties. - Biometrics 27: $857-871$.

Heemsbergen, D. A. et al. 2004. Biodiversity effects on soil processes explained by interspecific functional dissimilarity. - Science 306: 1019-1020.

Holmes, R. T. et al. 1979. Guild structure of the Hubbard Brook bird community: a multivariate approach. - Ecology 60: 512-520.

Hooper, D. U. et al. 2005. Effects of biodiversity on ecosystem functioning: a consensus of current knowledge. - Ecol Monogr 75: 3-35.

Hulot, F. D. et al. 2000. Functional diversity governs ecosystem response to nutrient enrichment. - Nature 405: 340-344.

393 Hutchinson, G. 1957. Concluding remarks. - Cold Spring Harbor Symp Quant Biol 22: 415-427.

394 Katrina, N. et al. 1985. Application of computer taxonomic techniques to the study of cutaneous propionibacteria and skin-surface lipid. - Arch Dermatol Res 278: 107-113.

Laliberté, E. and Legendre, P. 2010. A distance-based framework for measuring functional diversity from multiple traits. - Ecology 91: 299-305.

Laliberté, E. et al. 2008. Impacts of rangeland development on plant functional diversity, ecosystem processes and services, and resilience. - Global Land Project Newsletter 4: 4-6.

Lavorel, S. et al. 2008. Assessing functional diversity in the field-methodology matters! Functional Ecology 22: 134-147.

Lepš, J. et al. 2006. Quantifying and interpreting functional diversity of natural communities: practical considerations matter. - Preslia 78: 481-501.

Loreau, M. et al. 2001. Biodiversity and ecosystem functioning: current knowledge and future challenges. - Science 294: 804-808.

408 Maechler, M. et al. 2015. cluster: cluster analysis basics and extensions. in press.

409 Mason, N. et al. 2005. Functional richness, functional evenness and functional divergence: the primary components of functional diversity. - Oikos 111: 112-118.

411 McGill, B. J. 2015. Steering the trait bandwagon. - Dynamic Ecology in press. 
Mouchet, M. A. et al. 2010. Functional diversity measures: an overview of their redundancy and their ability to discriminate community assembly rules. - Functional Ecology 24: 867-876.

414 Pavoine, S. and Bonnan, M. B. 2011. Measuring biodiversity to explain community assembly: a unified approach. - Bio. Rev. 86: 792-812.

Pavoine, S. et al. 2009. On the challenge of treating various types of variables: application for improving the measurement of functional diversity. - Oikos 118: 391-402.

Petchey, O. L. and Gaston, K. J. 2002. Functional diversity (FD), species richness and community composition. - Ecol. Lett. 5: 402-411.

Petchey, O. L. and Gaston, K. J. 2006. Functional diversity: back to basics and looking forward. - Ecol. Lett. 9: 741-758.

Petchey, O. L. and Gaston, K. J. 2007. Dendrograms and measuring functional diversity. - Oikos

Petchey, O. L. et al. 2004. How do different measures of functional diversity perform? - Ecology 85: 847-857.

Petchey, O. L. et al. 2009. A functional guide to functional diversity measures. - In: Naeem, S. et al. (eds), Biodiversity, Ecosystem Functioning, and Human Wellbeing. ppp. 49-60.

Podani, J. 1999. Extending Gower's general coefficient of similarity to ordinal characters. Taxon 48: 331-340.

Podani, J. and Schmera, D. 2006. On dendrogram-based measures of functional diversity. Oikos 115: 179-185.

Podani, J. and Schmera, D. 2007. How should a dendrogram-based measure of functional diversity function? A rejoinder to Petchey and Gaston. - Oikos 116: 1427-1430.

Poos, M. S. et al. 2009. Functional-diversity indices can be driven by methodological choices and species richness. - Ecology 90: 341-347.

R Development Core Team 2015. R: a language and environment for statistical computing. in press.

Redding, D. W. et al. 2014. Measuring evolutionary isolation for conservation (WJ Murphy, Ed.). - PLoS ONE 9: e113490.

Rosenfeld, J. S. 2002. Functional redundancy in ecology and conservation. - Oikos 98: 156-162.

Schleuter, D. et al. 2010. A user's guide to functional diversity indices. - Ecol Monogr 80: 469484.

443 Schütz, C. and Schulze, C. H. 2015. Functional diversity of urban bird communities: effects of 444 landscape composition, green space area and vegetation cover. - Ecol. Evol. 5: 5230-5239. 
445 Sneath, P. H. A. and Sokal, R. R. 1973. Numerical Taxonomy. - W.H. Freeman and Company.

446 Stevens, R. D. and Tello, J. S. 2014. On the measurement of dimensionality of biodiversity. 447 Global Ecol Biogeogr 23: 1115-1125.

448 Thompson, K. et al. 2010. Little evidence for limiting similarity in a long-term study of a roadside plant community. - J. Ecol. 98: 480-487.

450 Tilman, D. 2001. Functional diversity. - In: Levin, S. A. (ed), Encyclopedia of Biodiversity. Academic Press, ppp. 109-120.

Tilman, D. et al. 1997. The influence of functional diversity and composition on ecosystem processes. - Science 277: 1300-1302.

454 Van Valkenburgh, B. 1988. Trophic diversity in past and present guilds of large predatory 455 mammals. - Paleobiol.: 155-173.

456 Villéger, S. et al. 2008. New multidimensional functional diversity indices for a multifaceted framework in functional ecology. - Ecology 89: 2290-2301.

Wright, J. P. et al. 2005. Conventional functional classification schemes underestimate the relationship with ecosystem functioning. - Ecol. Lett. 9: 111-120. 


\section{Tables}

\section{Table 1}

465 Different methods for calculating differences with ordinal traits. Let $\mathrm{X}$ be a matrix containing $m$

466 rows (taxa, typically species or individuals) and $n$ columns (traits, characters, variables) of

467 ordinal data. Further, let $i$ denote the index of a trait out of the $n$ traits considered. Let $x_{i j}$ be the

468 value taken by this trait for taxon $j$. Let $d_{i j k}$ be the difference between taxa $j$ and $k$ as measured by

469 trait $i$ with $D_{j k}$ being the total difference between the two taxa measured on all $n$ traits. Let $r_{i j}$ be

470 the rank of taxon's $j$ ordinal score among all scores of trait $i$. Let $T_{i j}$ be the number of taxa that

471 have the same rank for trait $i$ as taxon $j$ (including $j$ in this count). Let $T_{i, \max }$ be the number of

472 taxa that have the maximum $\operatorname{rank}\left(\max \left\{r_{i}\right\}\right)$ for trait $i$. 


\begin{tabular}{|c|c|c|c|}
\hline Equation & Source & Description & Equivalent function call in $\mathrm{R}$ \\
\hline $\begin{array}{l}d_{i j k}=0, \text { if } x_{i j}=x_{i k} \\
d_{i j k}=1, \text { if } x_{i j} \neq x_{i k}\end{array}$ & $\begin{array}{l}\text { (Gower 1971) } \\
\text { Equation 2b } \\
\text { (Sneath and } \\
\text { Sokal 1973) } \\
\text { pg. } 136\end{array}$ & $\begin{array}{l}\text { Categorical: } \\
\text { Ordered levels are treated as } \\
\text { unranked nominal categories }\end{array}$ & $\begin{array}{l}X 2<- \text { as.data. frame( apply(X, } \\
2 \text {, function( }(X) \text { factor }(X, \\
\text { ordered=F))); gowdis(X2) । } \\
\text { daisy }(X 2 \text {, metric= “gower") } \\
d<- \text { dist.ktab( } \\
\text { as.numeric(X), type }= \\
c(\text { (N”)); } d \wedge 2\end{array}$ \\
\hline$d_{i j k}=\frac{\left|x_{i j}-x_{i k}\right|}{\max \left\{x_{i}\right\}-\min \left\{x_{i}\right\}}$ & $\begin{array}{l}\text { (Gower 1971) } \\
\text { Equation 2c }\end{array}$ & $\begin{array}{l}\text { Continuous: } \\
\text { Ordinal scores treated like } \\
\text { continuous values with Manhattan } \\
\text { distance }\end{array}$ & 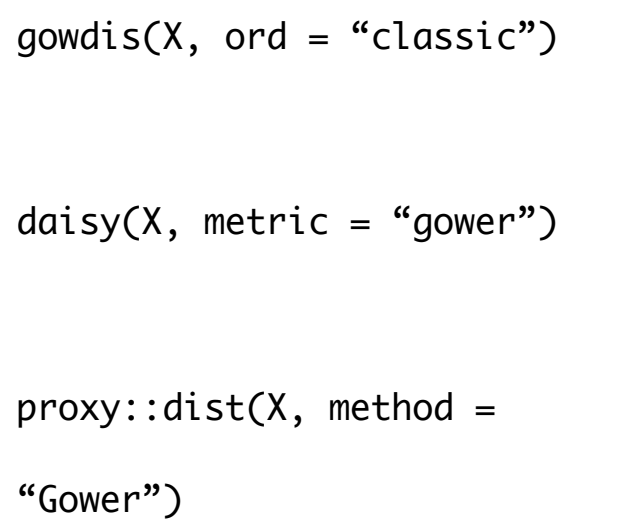 \\
\hline
\end{tabular}




\begin{tabular}{|c|c|c|c|}
\hline Equation & Source & Description & Equivalent function call in $\mathrm{R}$ \\
\hline$D_{j k}=\sqrt{\sum_{i=1}^{n}\left(\frac{x_{i j}-x_{i k}}{\max \left\{x_{i}\right\}-\min \left\{x_{i}\right\}}\right)^{2}}$ & This study & $\begin{array}{l}\text { Euclidean: } \\
\text { Ordinal scores treated like } \\
\text { continuous values with Euclidean } \\
\text { distance }\end{array}$ & $\begin{array}{l}X 2<- \text { as.matrix( data.frame( } \\
\operatorname{lapply}(X, \text { as.numeric }))) \\
\operatorname{dist}(\operatorname{apply}(X 2,2, \\
\text { function(x) } x /(\max (x)- \\
\min (x))))\end{array}$ \\
\hline $\begin{array}{l}\qquad d_{i j k}=0, \text { if } r_{i j}=r_{i k}, \\
\text { otherwise } \\
d_{i j k}=\frac{\left|r_{i j}-r_{i k}\right|-\frac{T_{i j}-1}{2}-\frac{T_{i k}-1}{2}}{\max \left\{r_{i}\right\}-\min \left\{r_{i}\right\}-\frac{T_{i, \max }-1}{2}-\frac{T_{i, \min }-1}{2}}\end{array}$ & $\begin{array}{l}\text { (Podani 1999) } \\
\text { Equation 2a-b }\end{array}$ & $\begin{array}{l}\text { Podani: } \\
\text { The minimum number of steps } \\
\text { (interchanges in the ordering of } \\
\text { taxa with neighboring ranks) } \\
\text { necessary to change one taxon's } \\
\text { rank into that of another, divided } \\
\text { by the possible maximum number } \\
\text { of steps }\end{array}$ & $\begin{array}{l}\text { gowdis(X, ord }=\text { "podani") } \\
d<- \text { dist.ktab( } \\
\text { as.numeric(X), type = c(" } 0 \text { "), } \\
\text { option = c("scaledBYrange"), } \\
\text { scann }=\mathrm{T}) ; 2 ; d^{\wedge} 2\end{array}$ \\
\hline
\end{tabular}




\begin{tabular}{|c|c|c|c|}
\hline Equation & Source & Description & Equivalent function call in $\mathrm{R}$ \\
\hline$d_{i j k}=\frac{\left|r_{i j}-r_{i k}\right|}{\max \left\{r_{i}\right\}-\min \left\{r_{i}\right\}}$ & $\begin{array}{l}\text { (Podani 1999) } \\
\text { Equation } 3\end{array}$ & $\begin{array}{l}\text { Metric: } \\
\text { Manhattan distance between ranks }\end{array}$ & $\begin{array}{l}\operatorname{gowdis}(X, \text { ord }=\text { "metric") } \\
d<- \text { dist.ktab( } \\
\text { as.numeric(X), type = c("0"), } \\
\text { option }=c(\text { ("scaledBYrange"), } \\
\text { scann = T); } 1 ; 2 ; d \wedge 2\end{array}$ \\
\hline$D_{j k}=\sqrt{\frac{1}{n} \sum_{i=1}^{n}\left(\frac{r_{i j}-r_{i k}}{\max \left\{r_{i}\right\}-\min \left\{r_{i}\right\}}\right)^{2}}$ & $\begin{array}{l}\text { (Pavoine et al. } \\
\text { 2009) } \\
\text { Equation } 3\end{array}$ & $\begin{array}{l}\text { Pavoine: } \\
\text { Euclidean distance between ranks } \\
\text { standardized by range }\end{array}$ & $\begin{array}{l}\text { dist.ktab( as. numeric }(X) \text {, } \\
\text { type }=c(\text { (O)"), option }= \\
c(\text { ("scaledBYrange") })\end{array}$ \\
\hline
\end{tabular}




\section{Table 2}

476 Popular continuous indices for measuring functional diversity.

\begin{tabular}{|c|c|c|c|c|c|}
\hline Index & Name & Definition & Low value & High Value & Source \\
\hline FD & $\begin{array}{l}\text { Functional } \\
\text { diversity }\end{array}$ & $\begin{array}{l}\text { Sum of branch lengths on } \\
\text { a functional dendrogram } \\
\text { needed to connect all taxa } \\
\text { in a community }\end{array}$ & $\begin{array}{l}\text { 0: Taxa are } \\
\text { identical in } \\
\text { functional traits }\end{array}$ & $\begin{array}{l}\text { Unbounded: } \\
\text { Taxa are far apart } \\
\text { in trait space }\end{array}$ & $\begin{array}{l}\text { (Petchey and } \\
\text { Gaston } \\
\text { 2007) }\end{array}$ \\
\hline FRic & $\begin{array}{l}\text { Functional } \\
\text { richness }\end{array}$ & $\begin{array}{l}\text { Volume of minimum } \\
\text { convex hull in trait space } \\
\text { enclosing all taxa in a } \\
\text { community }\end{array}$ & $\begin{array}{l}\text { 0: Taxa are } \\
\text { identical in } \\
\text { functional traits }\end{array}$ & $\begin{array}{l}\text { Unbounded: } \\
\text { Taxa are far apart } \\
\text { in trait space }\end{array}$ & $\begin{array}{l}\text { (Villéger et } \\
\text { al. 2008) }\end{array}$ \\
\hline FEve & $\begin{array}{l}\text { Functional } \\
\text { evenness }\end{array}$ & $\begin{array}{l}\text { Regularity of taxa along a } \\
\text { minimum spanning tree in } \\
\text { trait space }\end{array}$ & $\begin{array}{l}\text { 0: Taxa are } \\
\text { unevenly } \\
\text { distributed } \\
\text { throughout trait } \\
\text { space }\end{array}$ & $\begin{array}{l}\text { 1: Taxa are } \\
\text { evenly } \\
\text { distributed } \\
\text { throughout trait } \\
\text { space }\end{array}$ & $\begin{array}{l}\text { (Villéger et } \\
\text { al. 2008) }\end{array}$ \\
\hline FDiv & $\begin{array}{l}\text { Functional } \\
\text { divergence }\end{array}$ & $\begin{array}{l}\text { Average deviance from } \\
\text { the mean distance to the } \\
\text { centroid of trait space }\end{array}$ & $\begin{array}{l}\text { 0: Taxa are close } \\
\text { to center of trait } \\
\text { space }\end{array}$ & $\begin{array}{l}\text { 1: Taxa are at } \\
\text { trait extremes }\end{array}$ & $\begin{array}{l}\text { (Villéger et } \\
\text { al. 2008) }\end{array}$ \\
\hline FDis & $\begin{array}{l}\text { Functional } \\
\text { dispersal }\end{array}$ & $\begin{array}{l}\text { Mean distance to centroid } \\
\text { of trait space }\end{array}$ & $\begin{array}{l}0 \text { : Species are } \\
\text { clustered around } \\
\text { centroid }\end{array}$ & $\begin{array}{l}\text { Unbounded: } \\
\text { Taxa are far from } \\
\text { center of trait } \\
\text { space }\end{array}$ & $\begin{array}{l}\text { (Laliberté } \\
\text { and } \\
\text { Legendre } \\
\text { 2010) }\end{array}$ \\
\hline
\end{tabular}




\begin{tabular}{|l|l|l|l|l|l|}
\hline \multirow{2}{*}{ RaoQ } & Rao's & Half the average squared & & \\
functional distance & 0: Taxa are closely & Unbounded: & (Champely \\
& entropy & setween two randomly & packed in trait & Taxa are far from & and Chessel \\
& selected individuals with & space & each other & 2002) \\
& & & & \\
\hline
\end{tabular}




\section{Figures}

480

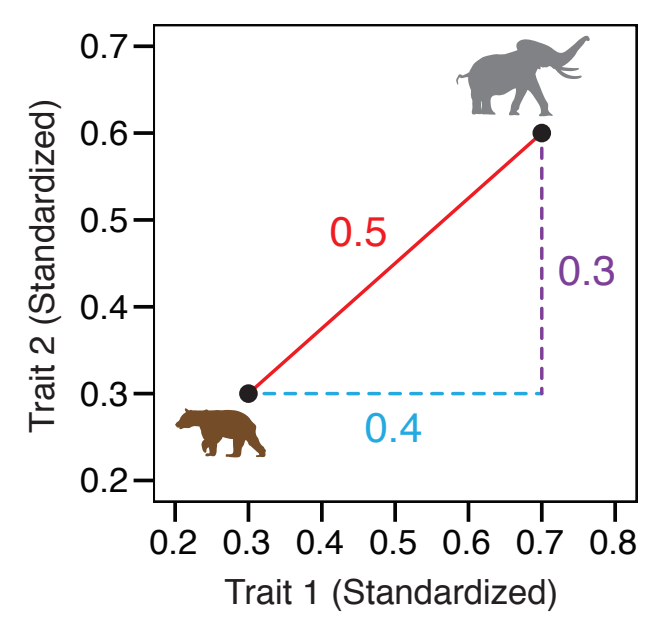

482 Figure 1

483 Given a niche space defined by $n$ functional traits (standardized by their respective ranges),

484 the difference between two taxa, say a bear and an elephant, is often conceptualized as the

485 Euclidean straight-line distance between them (i.e., 0.5). Gower's distance however, would

486 take the average of the difference in each functional trait (i.e. $(0.4+0.3) / 2=0.35)$.

487 


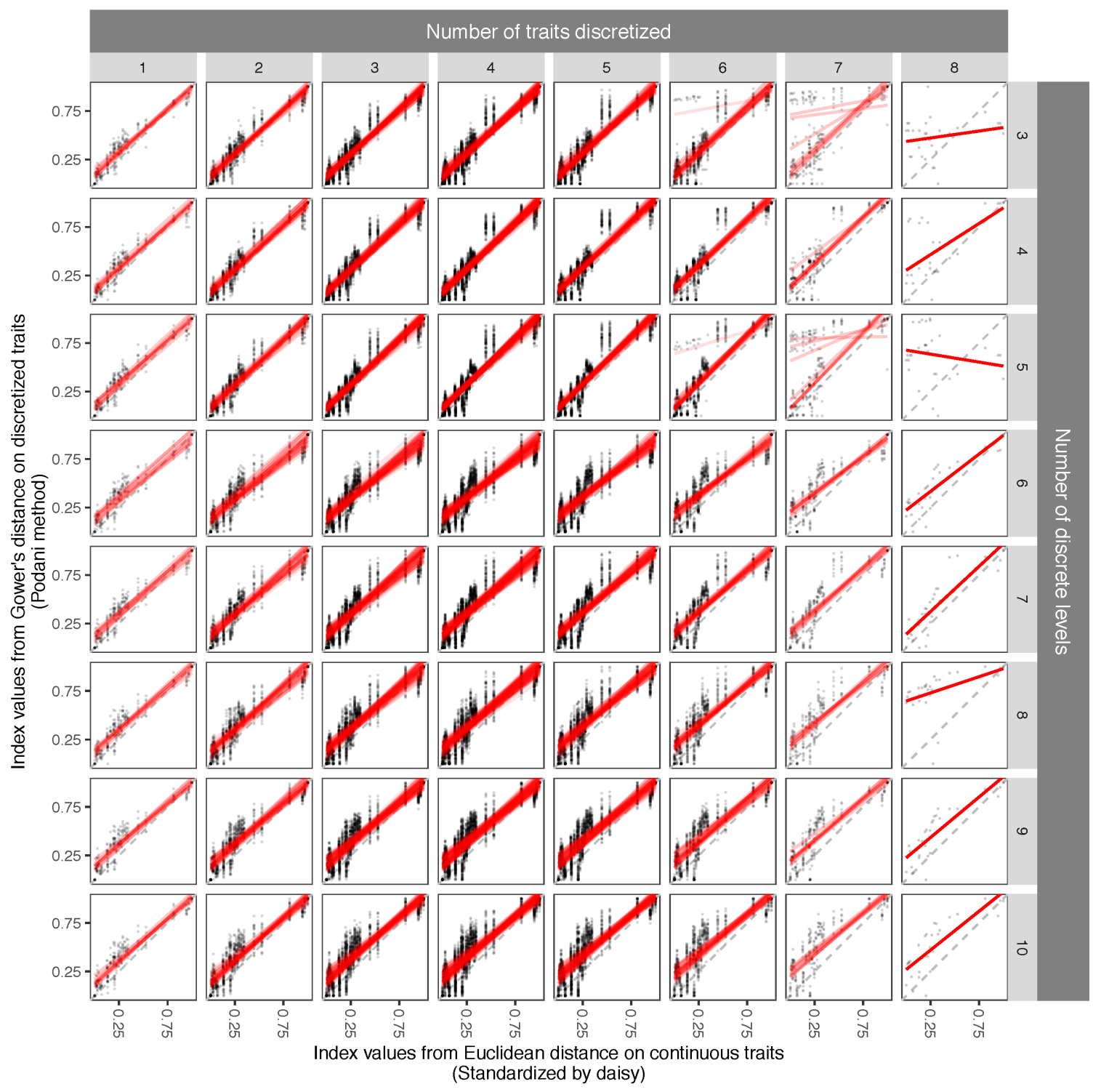

$489 \quad$ Figure 2

490 Relative values for functional evenness (FEve) differ between original and treatment versions

491 of the Tussock dataset. Each point represents the relative FEve of a plant community (in this

492 case, experimental plots in New Zealand). On the x-axis, relative FEve values were derived

493 from Euclidean distances between species with original continuous trait data standardized by

494 the daisy R function. On the y-axis, relative FEve values were derived from Gower's 
495 distances between species with trait data artificially discretized into ordered levels. The

496 Podani method was used to calculate Gower's distance on these treatment data. Dashed grey

497 guidelines mark a 1:1 relationship between the original and treatment data that points would

498 fall on if using discrete characters and different distance metrics had no effect on functional

499 diversity values. Red lines are linear model best fits to the data included only to guide the

500 eye. Most parameter combinations have more than one red line because there are multiple

501 ways to discretize a subset of eight traits (e.g. the two discretized traits could be the 1st and

502 2nd traits, or the 1 st and 3rd traits, or the 2nd and 3rd traits, etc.). 


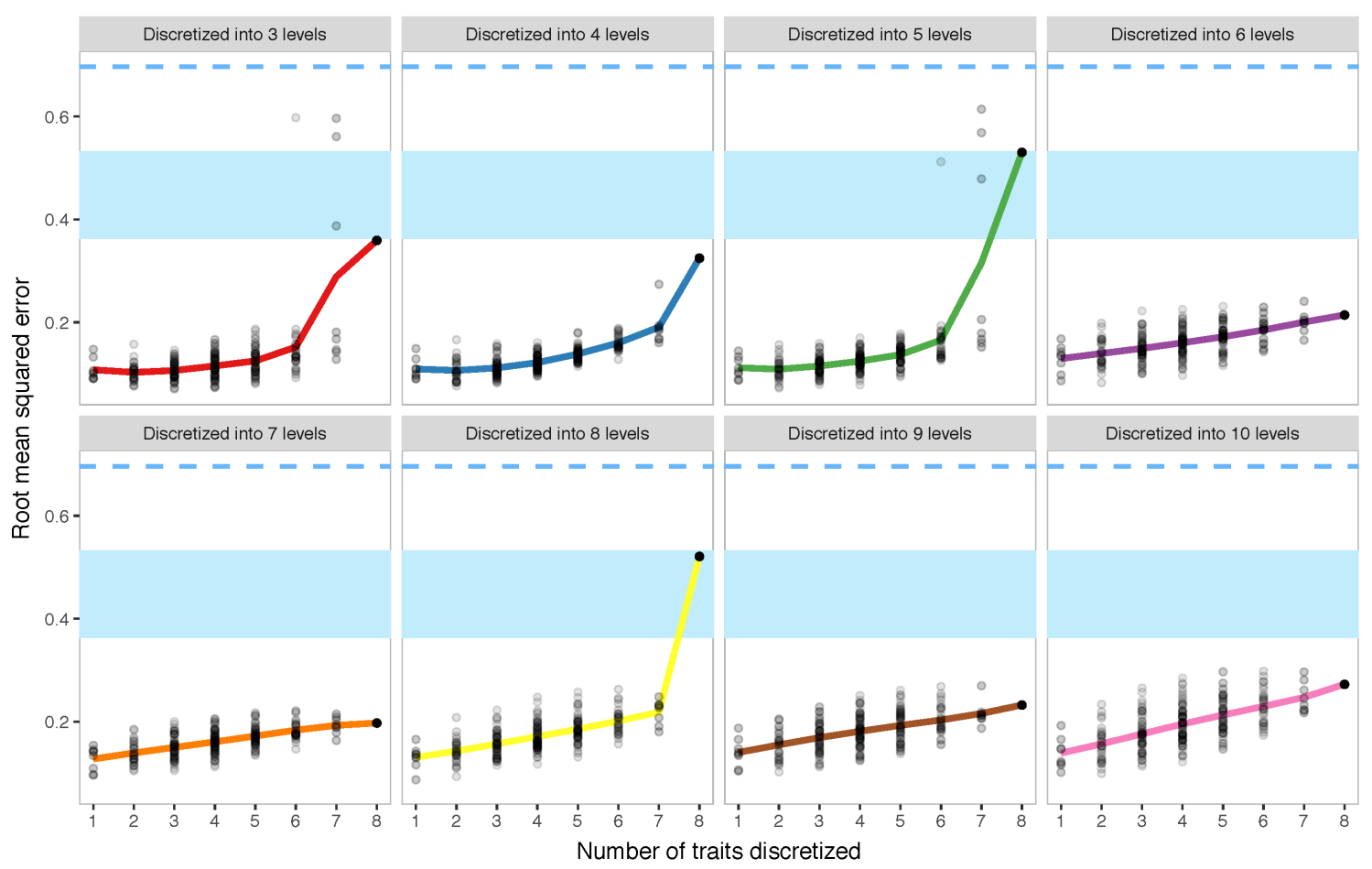

\section{$505 \quad$ Figure 3}

506 This figure summarizes Figure 2; each point represents the root mean squared error (RMSE)

507 between the original functional evenness (FEve) values and one parameter combination of

508 the treatment FEve values. Each colored line connects the means of each parameter

509 combination. The blue dashed line represents the RMSE of a perfect negative correlation to

510 the original FEve values and the blue box represents the $95 \%$ confidence interval of the

511 RMSE between the original FEve values and random values from a uniform distribution. 


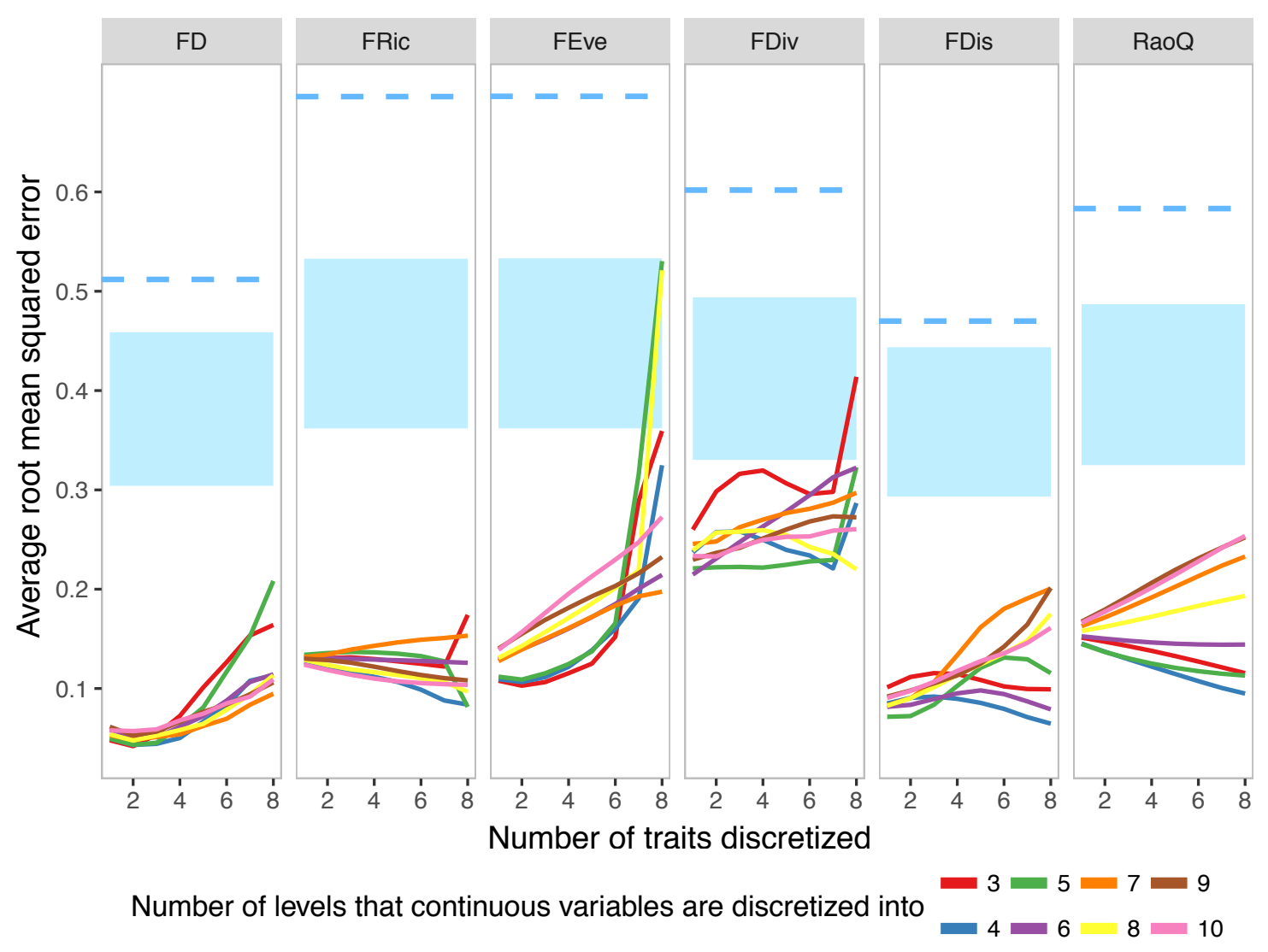

\section{$514 \quad$ Figure 4}

515 This figure summarizes the root mean squared errors (RMSE) between original functional

516 diversity index values generated from continuous data and the values obtained from different

517 parameter combinations of Gower's distance using the Podani method on artificially

518 discretized treatment data. Each colored line represents the average RMSE for a particular

519 parameter combination and matches the coloration used in Figure 3. The blue dashed lines

520 represent the RMSE of perfect negative correlations to the original functional diversity index

521 values and the blue boxes represent the $95 \%$ confidence intervals of the RMSE between the

522 original index values and random values from uniform distributions as in Figure 3. 


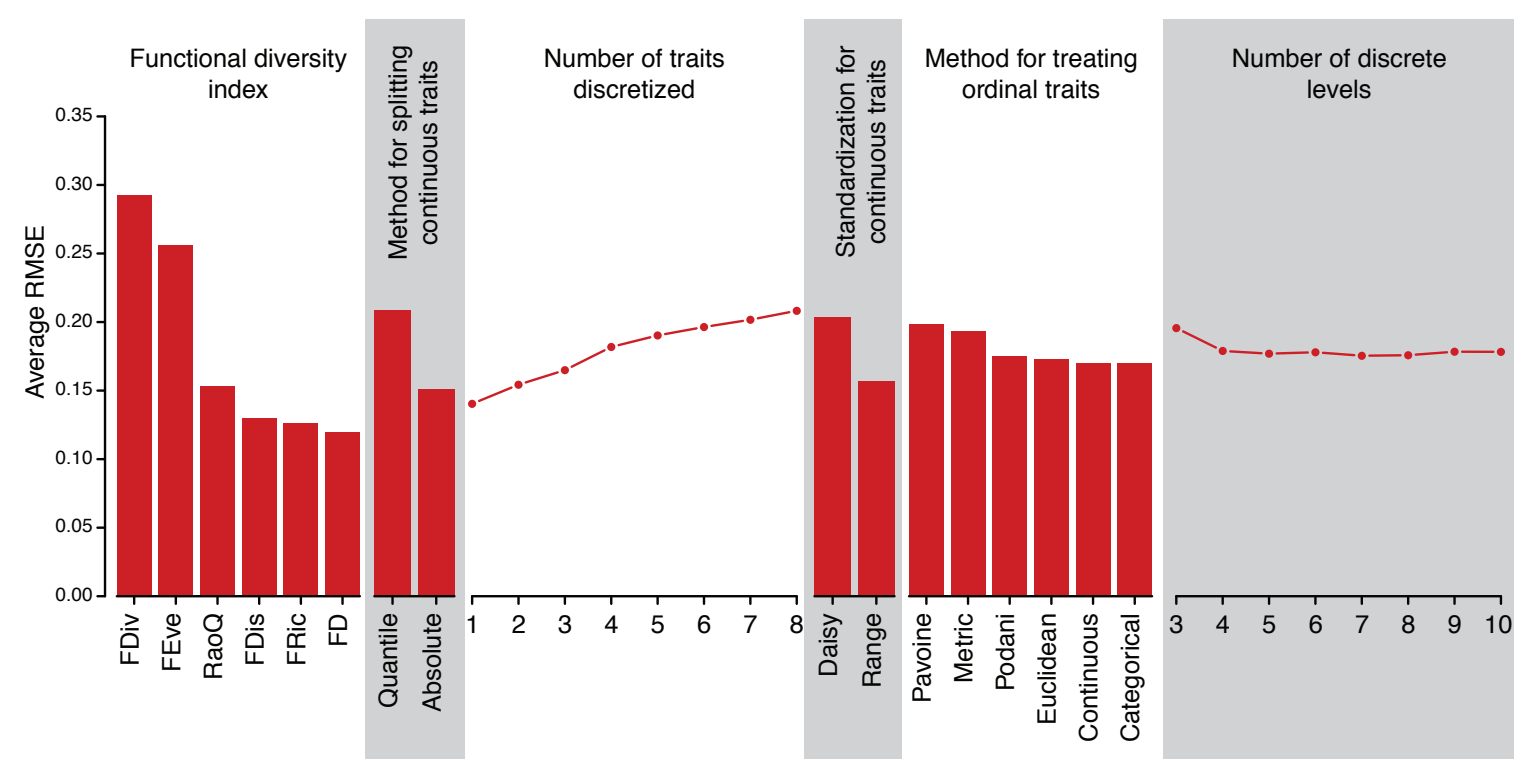

Figure 5

526 The importance of individual parameters in determining how closely treatment functional

527 diversity values match original index values in the Tussock dataset. The y-axis shows the

528 average root mean squared error (RMSE) between original and treatment values expected

529 from that parameter holding all other parameters constant. 


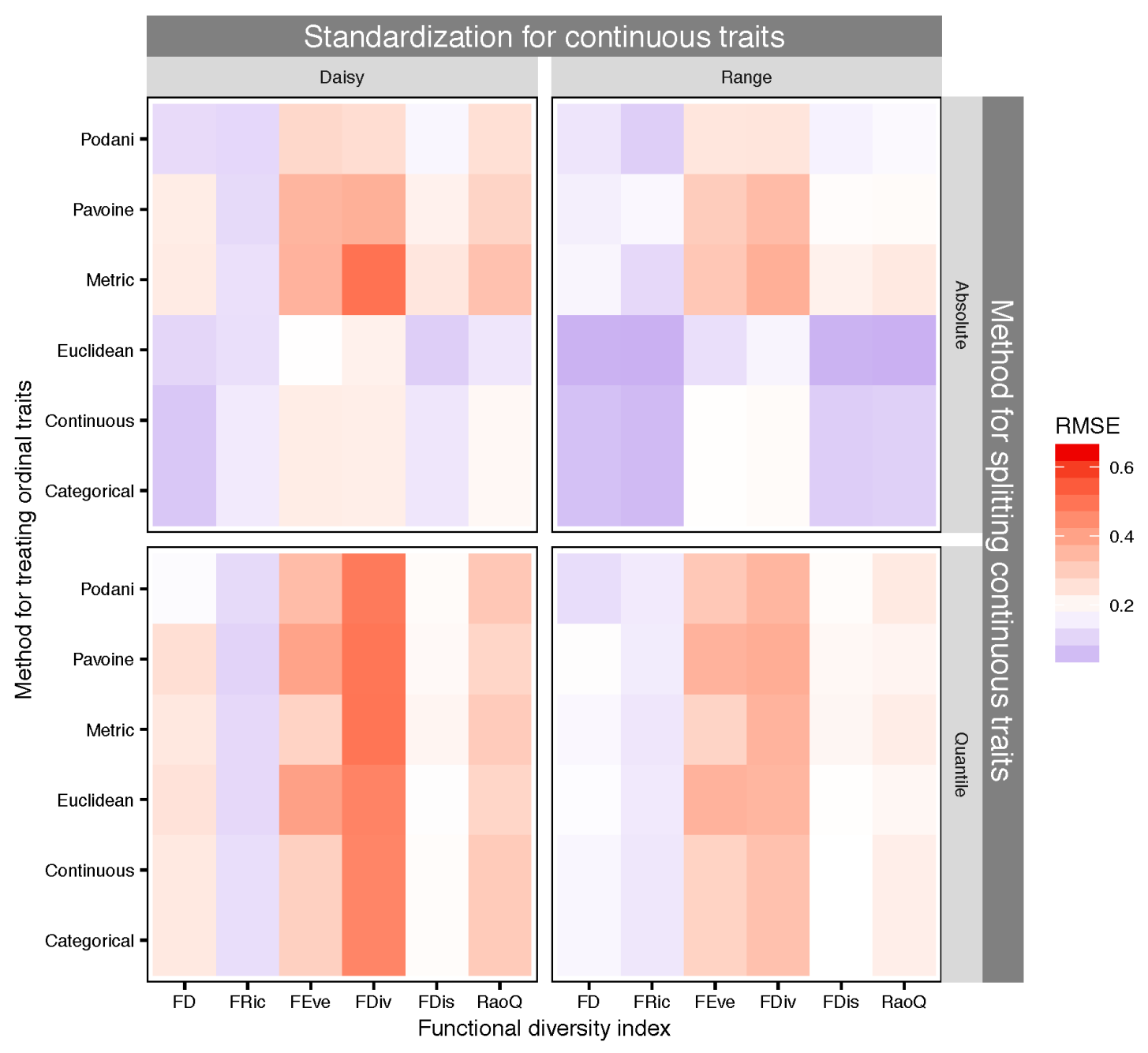

\section{Figure 6}

533 The importance of parameter combinations in determining how closely treatment functional

534 diversity values match original index values in the Tussock dataset. Colors designate the

535 average root mean squared error (RMSE) between the original continuous functional

536 diversity index values and the treatment values obtained from artificially discretized data.

537 Red hues show parameter combinations that give a worse RMSE than the average for all

538 parameter combinations (0.17) and blue hues show parameter combinations that return

539 RMSE better than average. 


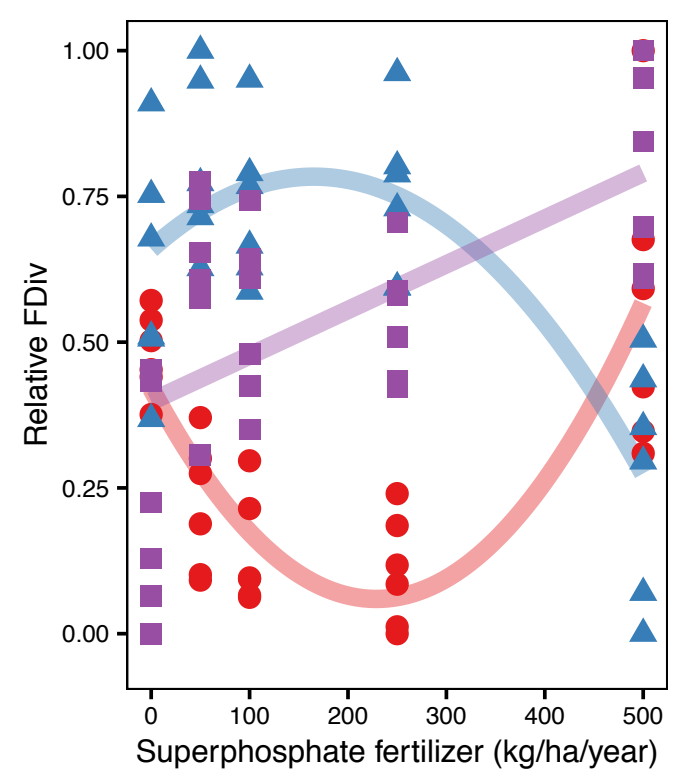

\section{$542 \quad$ Figure 7}

543 Small changes in methodology can have a large effect on perceived functional diversity

544 gradients. The graph shows the effect of different superphosphate loads on the relative

545 functional divergence (FDiv) of 30 real grassland plots in New Zealand from the Tussock

546 dataset. The red line and points show the relationship if original continuous data, daisy

547 standardization, and Euclidean distances are used. The blue line and points show the

548 relationship if two traits, leaf dry matter content and leaf phosphorous concentration, are

549 treated as ordinal variables, "Low", “Medium”, and "High" and Gower's distance (Podani

550 method) is used. The purple line and points show the relationship if all the parameters of the

551 blue line are followed except that leaf nitrogen concentration instead of leaf dry matter

552 content is treated as an ordinal trait, "Low", "Medium", and "High". 


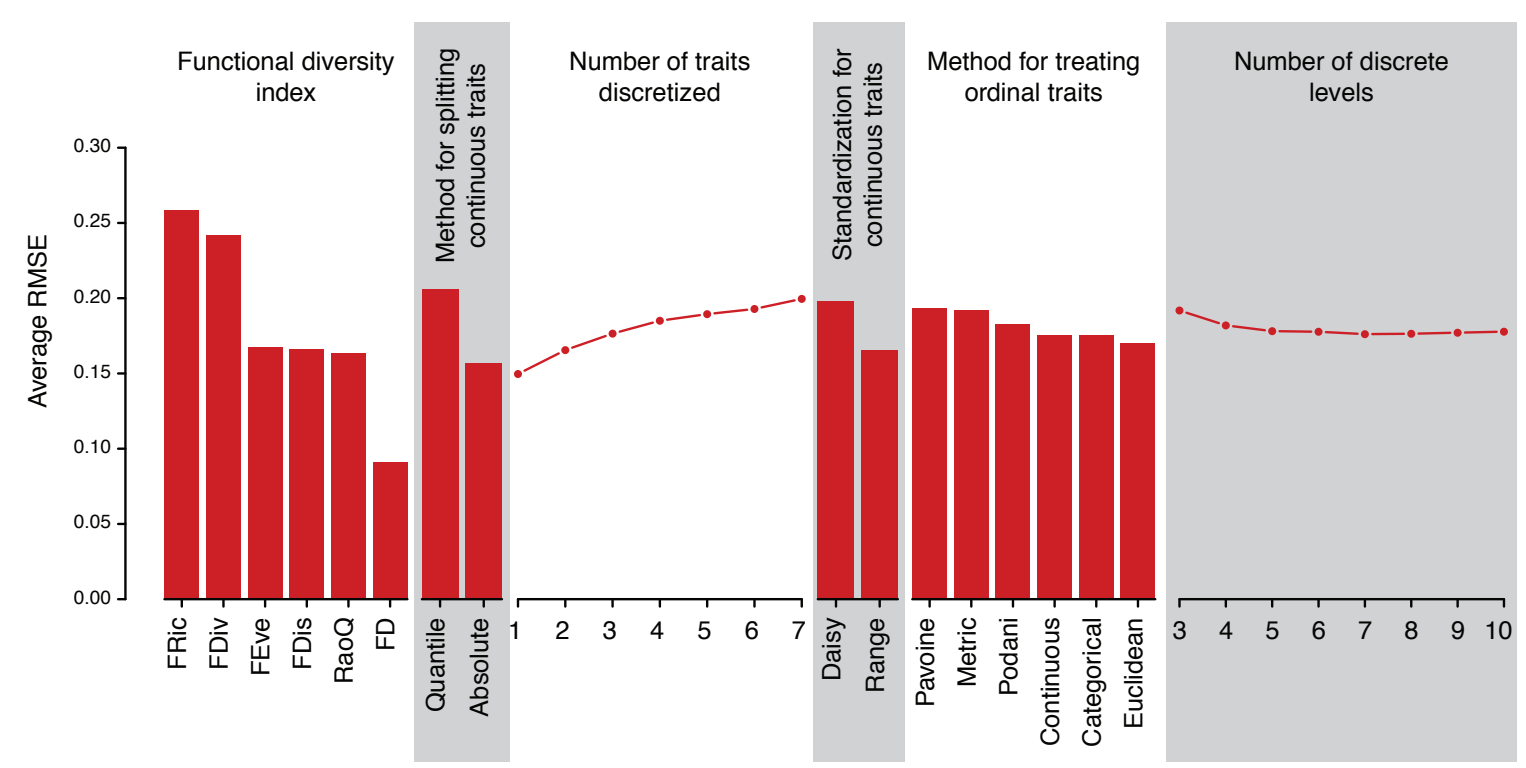

Figure A 1

556 The importance of individual parameters in determining how closely treatment functional

557 diversity values match original index values in the Roadside dataset. The y-axis shows the

558 average root mean squared error (RMSE) between original and treatment values expected

559 from that parameter holding all other parameters constant. 


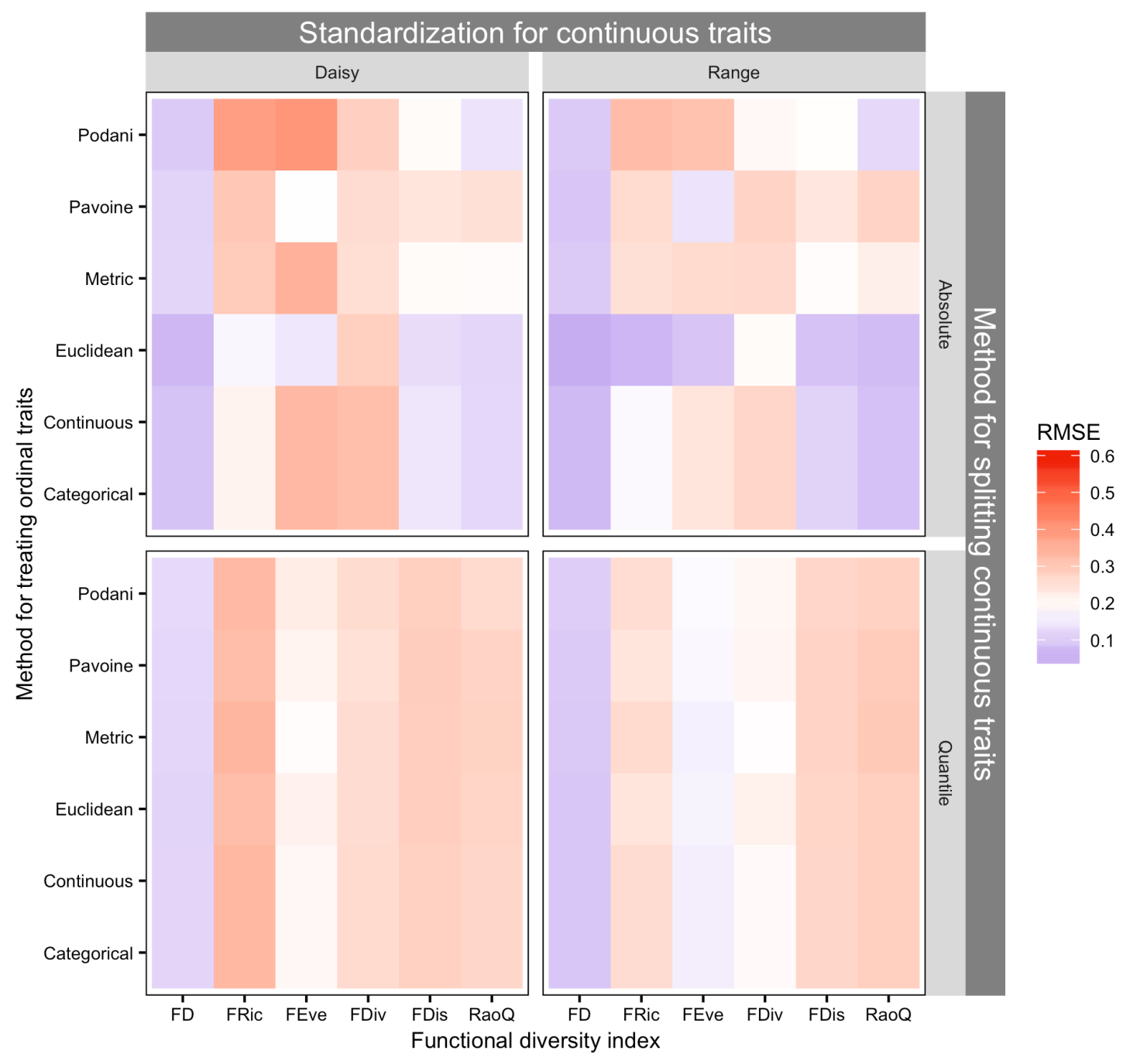

\section{Figure A 2}

563 The importance of parameter combinations in determining how closely treatment functional

564 diversity values match original index values in the Roadside dataset. Colors designate the

565 average root mean squared error (RMSE) between the original continuous functional

566 diversity index values and the treatment values obtained from artificially discretized data.

567 Red hues show parameter combinations that give a worse RMSE than the average for all

568 parameter combinations (0.18) and blue hues show parameter combinations that return

569 RMSE better than average. 